\title{
The Activity of Polymyxins against Dense Populations of Escherichia coli
}

\author{
By D. GREENWOOD* \\ Department of Bacteriology, St. Bartholomew's Hospital, London ECI $A{ }_{7} B E$
}

(Received 24 March 1975; revised I5 June 1975)

\begin{abstract}
SUMMARY
The activities of polymyxin B sulphate, colistin (polymyxin E) sulphate and their sulphomethyl derivatives were compared by continuous turbidimetric monitoring of dense cultures of an Escherichia coli strain exposed to these agents. Judged by the concentration of antibiotic which caused a rapid fall in opacity of the culture, polymyxin B sulphate and colistin sulphate had similar activities, but the sulphomethyl compounds differed considerably: sulphomyxin sodium induced lysis of the culture at a concentration four times that of the parent compound, whereas colistin sulphomethate sodium induced a delayed fall in opacity consistent with recruitment of activity as the inactive sulphomethyl derivative was broken down to the parent compound.

During overnight incubation, regrowth of cultures which had initially succumbed to polymyxin action occurred, apparently due to the selection of phenotypically resistant variants from within the population. In this way cultures could easily be adapted to growth in concentrations of antibiotic well above the conventionally-determined minimum inhibitory concentration. The comparative ease of adaptation was in the order: colistin sulphomethate $>$ sulphomyxin $>$ colistin sulphate $>$ polymyxin B sulphate.
\end{abstract}

\section{INTRODUCTION}

Although polymyxins have been in therapeutic use for many years a number of unanswered problems remain concerning their activity. In particular, conventional titrations of polymyxins are notoriously variable and the actual degree of activity of the sulphomethyl derivatives (which spontaneously break down to the parent compound) has remained a source of contention.

One of the reasons for the variability of conventional titrations of polymyxins is their susceptibility to changes in bacterial inoculum size (Bushby, I955; McCabe, Jackson \& Kozij, 1960). An approach to the problem of inoculum effects which has been found useful (Greenwood \& O'Grady, 1973) is to grow the bacteria in a photometric device and to add antibiotic at a standard point on the growth curve. This standardizes the inoculum and if the turbidity of the culture is continuously monitored, the kinetics of response to antibiotic during the whole of the incubation period may subsequently be observed, whereas conventional titrations are generally read at a single, arbitrarily chosen, point in time. This method of examination also has the advantage of employing a bacterial inoculum closer to that which may occur in actual infections.

Consequently, an investigation was initiated in which a turbidimetric method was used to compare the intrinsic activities of polymyxins B and E (colistin) and their sulphomethylated derivatives and to elucidate the nature of the variability of conventional titrations.

* Present address: Department of Microbiology, University of Nottingham, City Hospital, Nottingham NG5 IPH. 


\section{METHODS}

A strain of Escherichia coli (laboratory code ECSAI), originally derived from an infected urine, was used throughout.

Polymyxin B sulphate (Aerosporin) and sulphomyxin sodium (Thiosporin) were supplied by Burroughs Wellcome and Co.; colistin sulphate (Colomycin) and colistin sulphomethate sodium (Colomycin Injection) were from Pharmax Ltd. Polymyxin potencies are estimated in units, but because unit potencies of different polymyxins are not directly comparable and also because unit potencies of polymyxin B are given as 'not less than', we have preferred to use weighed amounts of the powders as provided by the manufacturers. The potencies of the preparations used, as stated by the manufacturers, were: polymyxin B sulphate, not less than 6500 units/mg; sulphomyxin sodium, 500000 units/vial (weight of contents not stated); colistin sulphate, I 9664 units/mg; colistin sulphomethate sodium, I 2773 units/mg. Suitable concentrations of antibiotic were prepared in sterile distilled water as required.

Growth medium was the complete broth of Greenwood \& O'Grady (I973). Turbidimetric measurements were made using the I2-channel, bacterial-growth monitoring device of Mackintosh et al. (1973). Cultures were grown from small inocula in the broth and antibiotic was added at a standard point in the exponential growth phase at a turbidity level ( $30 \%$ of maximum) equivalent to a viable count of about $5 \times 10^{7}$ organisms $/ \mathrm{ml}$.

To study adaptation, cultures were grown from small inocula in broth and antibiotic added at the standard turbidity level as above. Cultures succumbing to lysis, but subsequently growing again on continued incubation, were sub-inoculated, when fully regrown, into fresh broth containing antibiotic at the required concentration, and exposed to a further dose of antibiotic when growth again reached the standard point. A large inoculum ( $\mathrm{I} \mathrm{ml}$ of culture into $24 \mathrm{ml}$ of fresh, antibiotic-containing broth) was generally used for these transfers. Subcultures on solid medium were made to check that growth in antibiotic was not due to contaminant organisms.

Morphological changes induced by antibiotic were examined in the scanning electron microscope as described by Greenwood \& O'Grady (1969).

\section{RESULTS}

Sensitivity of E. coli to polymyxins in conventional titrations

Conventionally-estimated minimum inhibitory concentrations (m.i.c.) of the four polymyxins, in titrations employing a bacterial inoculum of about $10^{4}$ organisms $/ \mathrm{ml}$, were as follows $(\mu \mathrm{g} / \mathrm{ml}):$ polymyxin B sulphate, $\mathrm{I}$; sulphomyxin sodium, 4 ; colistin sulphate, 2; colistin sulphomethate sodium, 4. These represent typical results; a feature of these titrations was the variation in the results despite standardization of the inoculum. Anomalous results in which tubes showing bacterial growth occurred within a series of antibiotic dilutions where growth was inhibited were often encountered.

\section{Turbidimetric response of E. coli to polymyxins}

Typical continuous turbidity records obtained using serial doubling dilutions of two of the four polymyxins in the turbidimetric system are shown in Figs. I and 2. Polymyxin B sulphate (Fig. I) and colistin sulphate both induced a rapid fall in turbidity at a concentration of about $\mathrm{I} \mu \mathrm{g} / \mathrm{ml}$; sulphomyxin sodium induced a rapid fall in turbidity at a concentration of $4 \mu \mathrm{g} / \mathrm{ml}$; colistin sulphomethate sodium (Fig. 2) failed to induce a rapid fall in 


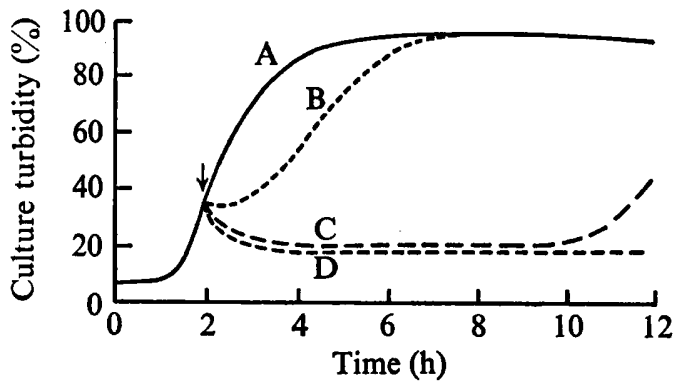

Fig. I

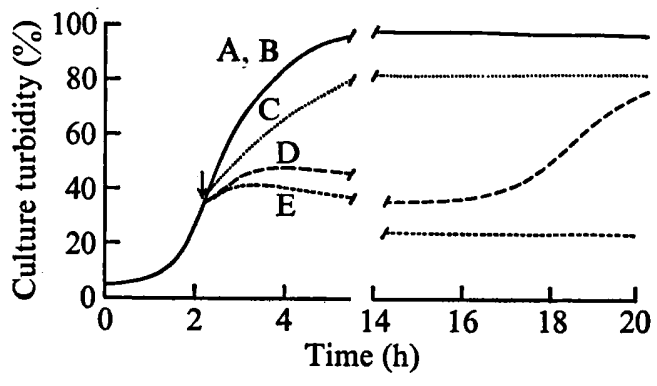

Fig. 2

Fig. I. Continuous turbidity records of E. coli strain ECSAI, showing the effect of polymyxin B sulphate (added at arrow) on exponentially-growing cultures. A, no antibiotic; B, I $\mu \mathrm{g} / \mathrm{ml} ; \mathrm{C}$, $2 \mu \mathrm{g} / \mathrm{ml} ; \mathrm{D}, 4 \mu \mathrm{g} / \mathrm{ml}$.

Fig. 2. Continuous turbidity records of $E$. coli strain ECSAI, showing the effect of colistin sulphomethate sodium (added at arrow) on exponentially-growing cultures. A, no antibiotic; $\mathrm{B}, 8 \mu \mathrm{g} / \mathrm{ml}$; $\mathrm{C}, \mathrm{I} 6 \mu \mathrm{g} / \mathrm{ml} ; \mathrm{D}, 32 \mu \mathrm{g} / \mathrm{ml} ; \mathrm{E}, 64 \mu \mathrm{g} / \mathrm{ml}$.

turbidity over the concentration range tested, although a slow delayed fall occurred over a prolonged period at concentrations of $32 \mu \mathrm{g} / \mathrm{ml}$ and above.

The pattern of regrowth of cultures which initially succumbed to polymyxin lysis may also be seen in Figs. I and 2. With polymyxin B sulphate no regrowth during the overnight incubation period occurred at concentrations above $2 \mu \mathrm{g} / \mathrm{ml}$, whereas with colistin sulphate recovery of the culture was observed at $8 \mu \mathrm{g} / \mathrm{ml}$. Regrowth of cultures exposed to sulphomyxin sodium also occurred at an antibiotic concentration of $8 \mu \mathrm{g} / \mathrm{ml}$, and following exposure to colistin sulphomethate sodium at a concentration of $32 \mu \mathrm{g} / \mathrm{ml}$. Again, these are typical values; twofold variations above and below these means were observed in different experiments with each of these antibiotics. Purity checks showed that the regrowth was not due to contamination.

\section{Morphological effects of polymyxin}

Morphological evidence that the fall in opacity consequent on the addition of antibiotic was due to bacterial lysis was obtained in the scanning electron microscope. Figure $3 a$ shows the normal appearance of the $E$. coli strain viewed in this way. The effects of $30 \mathrm{~min}$ exposure to Io $\mu \mathrm{g}$ polymyxin B sulphate/ml (added at the same point in the growth curve as in the turbidimetric experiments) is seen in Fig. $3 b$. This picture shows the apparent progression of the lesion induced in susceptible cells, from unaffected (Fig. $3 b, \mathrm{~A}$ ) to complete collapse (Fig. $3 b$, E). The effect of polymyxin B sulphate on Pseudomonas aeruginosa was also studied. A greater proportion of these bacteria collapsed after $\mathrm{I} h$ exposure to Io $\mu \mathrm{g}$ polymyxin B sulphate $/ \mathrm{ml}$ than $\operatorname{did} E$. coli.

\section{Adaptation to polymyxins}

Cultures exposed in the late-exponential growth phase to a maximum concentration of $4 \mu \mathrm{g}$ polymyxin B sulphate $/ \mathrm{ml}, 8 \mu \mathrm{g}$ colistin sulphate or sulphomyxin sodium $/ \mathrm{ml}$, and $32 \mu \mathrm{g}$ colistin sulphomethate sodium $/ \mathrm{ml}$ regrew during the $24 \mathrm{~h}$ incubation period. On subinoculating $\mathrm{I} \mathrm{ml}$ of the regrown cultures into fresh broth containing the same antibiotic concentration, uninterrupted growth occurred at a rate equivalent to that of a control culture which had not been exposed to antibiotic. On addition of more antibiotic to such cultures when growth had again reached the standard turbidity level, lysis again ensued (but was much more gradual than that seen with a previously-unexposed culture) and 

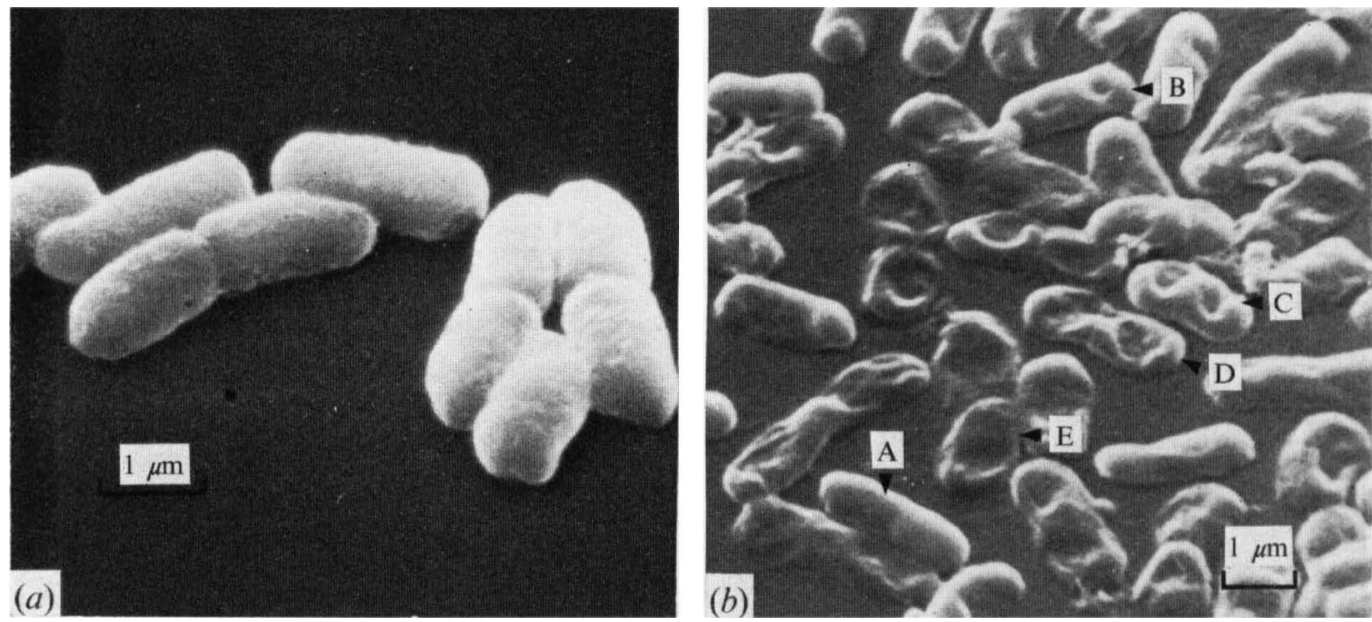

Fig. 3. Scanning electron micrographs of $E$. coli strain ECSAI. (a) Normal cells; $(b)$ same strain exposed to $10 \mu \mathrm{g}$ polymyxin B sulphate $/ \mathrm{ml}$ for $30 \mathrm{~min}$. A to E show the apparent progression of the lesion induced, from unaffected (A) to total collapse (E).

recovery was again observed during the overnight incubation period. The maximum concentration of additional antibiotic allowing regrowth of this first subculture was $(\mu \mathrm{g} / \mathrm{ml})$ : polymyxin B sulphate, 20; colistin sulphate, 44; sulphomyxin sodium, 64; colistin sulphomethate sodium, $>72$. These figures were obtained by using incremental steps of $4 \mu \mathrm{g} / \mathrm{ml}$ for the polymyxin sulphates and $8 \mu \mathrm{g} / \mathrm{ml}$ for the sulphomethylpolymyxins.

\section{Morphological effects of additional antibiotic on adapted cultures}

The morphological changes accompanying the delay in lysis of the adapted cultures when challenged with additional antibiotic were examined in the scanning electron microscope. A culture adapted to grow in $4 \mu \mathrm{g}$ polymyxin B sulphate $/ \mathrm{ml}$ was further exposed to an additional $24 \mu \mathrm{g} / \mathrm{ml}$. After $30 \mathrm{~min}$ exposure to $28 \mu \mathrm{g} / \mathrm{ml}$, a certain amount of cell collapse was detectable in some bacteria (Fig. $4 a$ ). Even after $2 \mathrm{~h}$, when many cells had totally succumbed to the antibiotic effect, morphologically normal cells were still present (Fig. $4 b$ ). An occasional but striking finding was the presence of spherical attachments to affected cells (Fig. $4 c$ ) reminiscent of the sphaeroplasts which emerge from Gram-negative bacilli exposed to high concentrations of $\beta$-lactam antibiotics (Greenwood \& O'Grady, I973).

\section{Further adaptation}

Although cultures regrowing after a single exposure to comparatively low concentrations of polymyxins grew freely on subcultivation into antibiotic-containing broth, further exposure of such cultures to higher concentrations of antibiotic induced an anomalous response (Fig. 5). This culture had ostensibly been adapted, by a single passage, to grow in $24 \mu \mathrm{g}$ polymyxin B sulphate $/ \mathrm{ml}$, in that growth occurred at this antibiotic concentration during the overnight incubation period. On sub-inoculation into fresh broth containing a series of concentrations of antibiotic it was found, however, that growth at a rate equivalent to that of a control culture in antibiotic-free broth occurred only at an antibiotic concentration of $4 \mu \mathrm{g} / \mathrm{ml}$. At slightly higher concentrations ( 8 and $\mathrm{I} 2 \mu \mathrm{g} / \mathrm{ml}$ ) uninterrupted but rather slower growth occurred; at $\mathrm{I} 6 \mu \mathrm{g} / \mathrm{ml}$ a biphasic response was induced, while at higher 

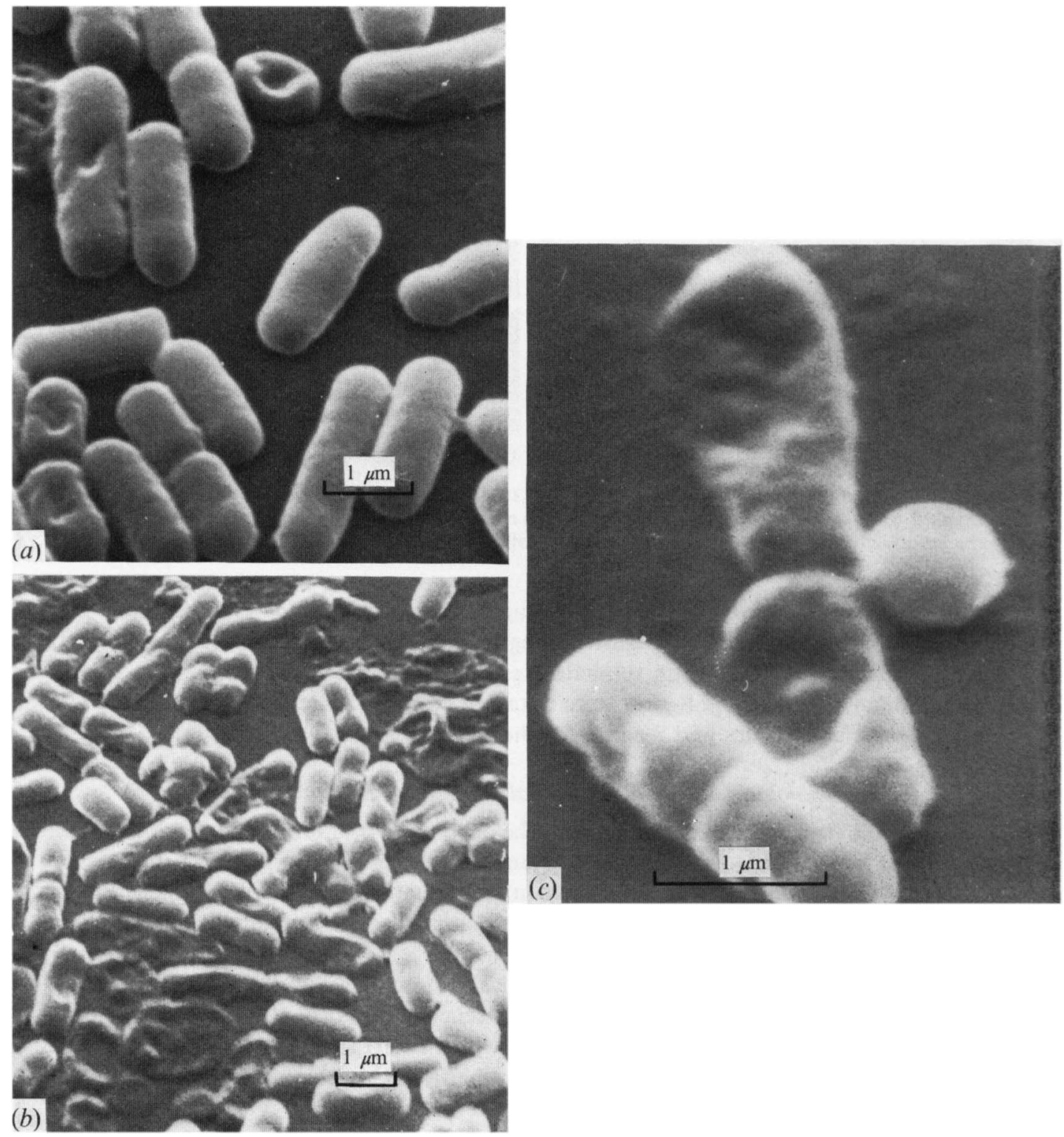

Fig. 4. Scanning electron micrographs of E. coli strain ECSAI, adapted to grow in $4 \mu$ g polymyxin B sulphate $/ \mathrm{ml}$, exposed to a further $24 \mu \mathrm{g}$ polymyxin B sulphate/ml for $(a) 30 \mathrm{~min}$ and $(b$ and $c$ ) $2 \mathrm{~h}$. The spherical attachment found on some collapsed cells is shown in $(c)$.

concentrations the culture appeared to grow during the first hour, but its turbidity subsequently declined again on continued incubation and uninterrupted regrowth occurred only after about $9 \mathrm{~h}$. The culture which eventually regrew in $24 \mu \mathrm{g}$ polymyxin $/ \mathrm{ml}$ in this experiment, having been grown twice in that concentration of polymyxin, was found to be completely adapted when reinoculated into fresh broth containing $24 \mu \mathrm{g}$ polymyxin $/ \mathrm{ml}$. 


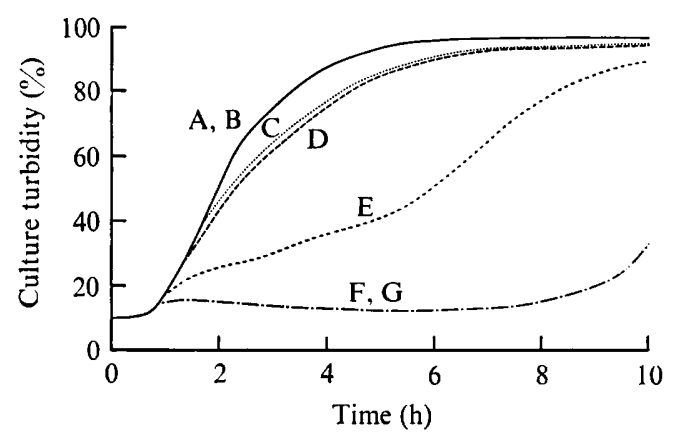

Fig. 5

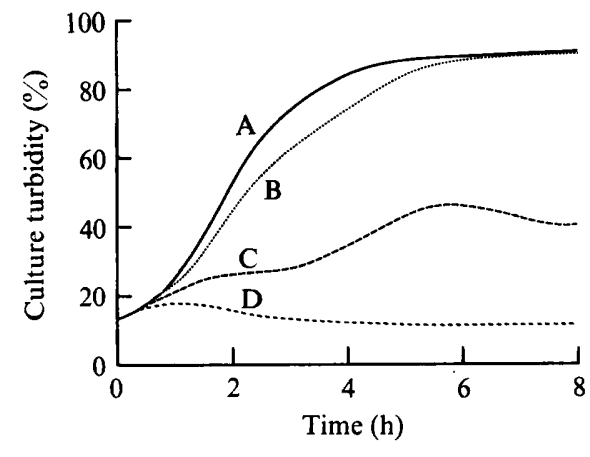

Fig. 6

Fig. 5. Continuous turbidity records of $E$. coli strain ECSAI, showing the ability of ostensibly adapted cultures to grow in different concentrations of polymyxin. One ml of a culture after exposure to $24 \mu \mathrm{g}$ polymyxin B sulphate $/ \mathrm{ml}$ was added at time 0 to $24 \mathrm{ml}$ of fresh broth containing polymyxin $\mathrm{B}$ sulphate at the following concentrations $(\mu \mathrm{g} / \mathrm{ml}): \mathrm{A}, 0 ; \mathrm{B}, 4 ; \mathrm{C}, 8 ; \mathrm{D}, \mathrm{I} 2 ; \mathrm{E}, \mathrm{I} 6$; F, 20; G, 24 .

Fig. 6. Continuous turbidity records of $E$. coli strain ECSAI, showing the susceptibility of a culture completely adapted to $24 \mu \mathrm{g}$ polymyxin B sulphate $/ \mathrm{ml}$ by passaging twice in that concentration of antibiotic. One $\mathrm{ml}$ of culture growing in $24 \mu \mathrm{g}$ polymyxin B sulphate $/ \mathrm{ml}$ was added at time 0 to fresh broth containing the antibiotic at the following concentrations $(\mu \mathrm{g} / \mathrm{ml}): \mathrm{A}, \mathrm{I} 6 ; \mathrm{B}, 24 ; \mathrm{C}, 32$; D, 40 .

\section{Stability of adaptation to polymyxin}

A culture was completely adapted to grow in $24 \mu \mathrm{g}$ polymyxin B sulphate/ml by passaging it twice through this concentration of antibiotic (see above). As soon as growth was complete (i.e. as it entered the stationary phase) the culture growing in this concentration of antibiotic was sub-inoculated into fresh broth containing graded concentrations of antibiotic (Fig. 6). The culture grew in $24 \mu \mathrm{g}$ polymyxin/ml, was partially susceptible to $32 \mu \mathrm{g} /$ $\mathrm{ml}$ and fully susceptible to $40 \mu \mathrm{g} / \mathrm{ml}$. When the experiment was repeated the following day, using as inoculum the same original culture (which by then had been in the stationary phase for $24 \mathrm{~h}$, but still in broth containing $24 \mu \mathrm{g}$ polymyxin/ml), the growth pattern was altered. Growth occurred in $8 \mu \mathrm{g}$ polymyxin/ml, was slightly delayed in $16 \mu \mathrm{g} / \mathrm{ml}$, and substantially delayed in antibiotic concentrations of 24 and $32 \mu \mathrm{g} / \mathrm{ml}$. Repeating the experiment once more with the same inoculum after it had remained in contact with polymyxin in the stationary phase for 4 days revealed a continuing increase in susceptibility: immediate growth occurred at a polymyxin concentration of $4 \mu \mathrm{g} / \mathrm{ml}$, delayed growth at $8 \mu \mathrm{g} / \mathrm{ml}$, very delayed growth at $16 \mu \mathrm{g} / \mathrm{ml}$ and no growth, during the period of observation, at a polymyxin concentration of $24 \mu \mathrm{g} / \mathrm{ml}$.

\section{Cross-adaptation between polymyxins}

Cultures adapted to grow in either of the sulphomethylpolymyxins showed cross-adaptation to the other, but remained fully susceptible to the two polymyxin sulphates. Cultures adapted to colistin sulphate showed cross-adaptation to the two sulphomethyl derivatives, but not to polymyxin B sulphate. Cultures adapted to polymyxin B sulphate showed crossadaptation to colistin sulphate and both of the sulphomethyl derivatives. 


\section{DISCUSSION}

Conventional tests of bacterial sensitivity to polymyxins are notoriously variable because of inoculum effects, inexact end-points and, in the case of diffusion tests, the slow diffusion rate characteristic of this group of agents (Bushby, 1955; McCabe et al. 1960). Continuous turbidimetric monitoring of bacterial cultures exposed to polymyxins has also given variable results, and as all other conditions of exposure were kept constant the differences may be attributed to heterogeneity of susceptibility between bacterial populations. Following the response of the cultures throughout the incubation period has revealed that polymyxin $B$ sulphate and colistin sulphate have similar activities, as judged by the concentration of antibiotic which caused rapid lysis of the culture. Dense cultures were able to recover to some extent from the antibiotic effect, in that they regrew during the overnight incubation period at concentrations of antibiotic which initially caused bacterial lysis. This regrowth occurred at higher concentrations of colistin sulphate than polymyxin B sulphate.

There has been controversy in the past about the relative activities of sulphomethylpolymyxins; since they spontaneously degrade to less completely sulphomethylated compounds, it is always unclear to what extent experimental results are influenced by alteration in activity during the incubation period (Barnett, Bushby \& Wilkinson, 1964; Beveridge \& Martin, 1967). Results obtained by continuous turbidimetric monitoring show that sulphomyxin sodium is very active, in that rapid lysis followed exposure to quite modest amounts of the agent before spontaneous breakdown had time to occur. Colistin sulphomethate sodium, on the other hand, appeared very inactive according to this criterion, but a slow and prolonged fall in opacity occurred at high antibiotic concentration, suggesting that the activity may indeed be due to breakdown to a more active product. A possible reason for this difference in activity of the two sulphomethylpolymyxins is that sulphomyxin contains a significant amount of the mono-substituted compound and this retains a major part of the parent activity. Sulphomethylpolymyxins are known to be heterogeneous mixtures of the mono-, di-, tri-, tetra- and penta-substituted compounds (Barnett et al. I964; Beveridge \& Martin, I967), and electrophoresis studies by both Barnett et al. (1964) and Beveridge \& Martin (I967) appear to show that sulphomyxin contains substantially more of what is taken to be mono-substituted derivative than does colistin sulphomethate. The present results show that sulphomyxin has about one-quarter the activity of its parent, whereas the activity of colistin sulphomethate is less than $\frac{1}{64}$ th that of the parent compound. As this difference is not shown in conventional titrations, the inoculum effect must be particularly marked with colistin sulphomethate.

Adaptive resistance to polymyxin was described and investigated soon after the antibiotic became available (Jawetz \& Coleman, 1949), but the rapidity with which facultatively resistant bacteria can be selected from dense bacterial populations does not appear to have been appreciated at that time or in subsequent studies (Schwartz, 1964). The differential capacity of bacteria to adapt to polymyxin sulphates and their sulphomethyl derivatives appears to be undocumented.

The ease with which this form of phenotypic resistance can be induced in the case of polymyxins is unusual. As it is markedly affected by population size, it probably reflects a heterogeneity of response within the bacterial population. Polymyxins are thought to act in a similar manner to cationic detergents by binding to membrane phospholipids and thus increasing the permeability of the cell membrane (Sebek, 1967; Gale et al. 1972). This suggests that, as the polymyxins act directly on a pre-formed structure rather than imposing a biochemical block in a metabolic process of the cell, phenotypically 'resistant' cells pre- 
exist in the population and a wide variation in phospholipid composition occurs among $E$. coli cells in an ostensibly homogeneous culture. The process by which the pre-formed 'resistant' cells are selected from such a population was well described by Yudkin (1953).

An interesting finding of this study was that 'complete' adaptation (in that uninterrupted growth occurred upon subculture into antibiotic-containing broth) was unstable in the stationary phase, even when the culture remained exposed to antibiotic. Stationary-phase cultures of $E$. coli are known to contain much larger amounts of cyclopropane fatty acids than is the case with cells harvested during exponential growth (Marr \& Ingraham, 1962), and resistance of $K$. pneumoniae to polymyxin is associated with markedly reduced quantities of cyclopropane fatty acids (Dunnick \& O'Leary, 1970). If such membrane changes occur during the stationary phase, then the susceptibility to antibiotic may increase concurrently. If this is so, why do the cells not become susceptible to the antibiotic which remains present during the stationary-phase incubation? One explanation would be that the polymyxin becomes inactive during this period. This would also explain the apparent, but spurious, adaptation seen when cultures adapted to intermediate concentrations of polymyxin were re-exposed to higher antibiotic concentrations. Polymyxin sulphate is inherently very stable (Jawetz \& Coleman, 1949; Schwartz, I964) but it is possible that the bacteria modify the antibiotic in some way. Evidence for such polymyxin inactivation has recently been presented by Kauffmann (1974), who ascribed the inoculum effect to neutralization of polymyxin by membrane phospholipids. The stability of the regrowing population to the addition of more antibiotic suggests that adaptive resistance is an equally important explanation of the inoculum phenomenon. The 'spurious' adaptation to high concentrations of polymyxins cannot entirely be ascribed to polymyxin inactivation, as such 'adaptation' became complete after one further sub-culture into antibiotic-containing broth. Whatever the reason for the apparent loss of activity, the antibiotic evidently remains bound to the bacteria and must be displaced before fresh antibiotic can exert its antibacterial effect. This is suggested by the initial rise in turbidity of incompletely-adapted cultures following sub-inoculation into broth containing antibiotic at a level to which the organisms were still susceptible, and is supported by the morphological changes seen in the scanning electron microscope, which revealed that lysis of adapted bacteria was much more gradual than that of organisms previously unexposed to antibiotic.

The phenomenon of adaptive resistance has been neglected to the extent that a recent authoritative review of the mechanisms of antibiotic resistance to antibiotics (Benveniste \& Davies, 1973) failed to mention it. With most antibiotics, adaptation to significant degrees of resistance takes numerous subcultures in highly artificial circumstances in the laboratory and it is probably irrelevant to the use of drugs in therapeutic practice. The ease with which $E$. coli has been shown to adapt to quite high levels of polymyxins in the present study raises the question of whether the phenomenon may have some therapeutic significance with this group of antibiotics.

\section{REFERENCES}

Barnett, M., Bushby, S. R. M. \& Wilkinson, S. (1964). Sodium sulphomethyl derivatives of polymyxins. British Journal of Pharmacology 23, 552-574.

Benveniste, R. \& Davies, J. (I973). Mechanisms of antibiotic resistance in bacteria. Annual Review of Biochemistry 42, 47I-506.

Beveridge, E. G. \& MARTin, A. J. (1967). Sodium sulphomethyl derivatives of polymyxins. British Journal of Pharmacology and Chemotherapy 29, 125-135.

Bushby, S. R. M. (1955). Sensitivity tests for the polymyxins. Journal of Clinical Pathology 8, I $20-$ I 22.

DunNick, J. K. \& O'LeARY, W. M. (I970). Correlation of bacterial lipid composition with antibiotic resistance. Journal of Bacteriology ror, 892-900. 
Gale, E. F., Cundliffe, E., Reynolds, P. E., Richmond, M. H. \& Waring, M. J. (i972). The Molecular Basis of Antibiotic Action. London: John Wiley.

Greenwood, D. \& O'Grady, F. (1969). Antibotic-induced surface changes in microorganisms demonstrated by scanning electron microscopy. Science, New York 163, 1076-1078.

Greenwood, D. \& O'Grady, F. (1973). Comparison of the responses of Escherichia coli and Proteus mirabilis to seven beta-lactam antibiotics. Journal of Infectious Diseases 128, 2 I I-222.

JAWETz, E. \& Coleman, V. R. (1949). Laboratory and clinical observations on Aerosporin (polymyxin B). Journal of Laboratory and Clinical Medicine 34, 75I-760.

Kauffmann, J. (1974). Méchanisme de l'effet inoculum (effet de population) chez Pseudomonas aeruginosa sur milieu additionné de polymyxine. Comptes rendus hebdomadaire des séances de l'Académie des sciences 279D, 207-209.

Mackintosh, I. P., O'Grady, F., Greenwood, D., Watson, B. W., Crichton, T. C., Piper, R. \& Ferrer, A. (1973). A twelve channel bacterial growth monitoring system. Biomedical Engineering 8, 514-515 and 526.

MARr, A. G. \& Ingraham, J. L. (I962). Effect of temperature on the composition of fatty-acids in Escherichia coli. Journal of Bacteriology 84, I 260-1 267.

MCCABE, W. R., JACKson, G. G. \& KozIJ, V. M. (1960). Clinical and laboratory observations on the use of colistin in infections by Gram-negative bacilli. In Antibiotics Annual, 1959-1960, pp. 80-88. Edited by F. Marti-Ibanez. New York: Antibiotica.

SCHWARTZ, B. S. (I964). The polypeptides of the polymyxin group. In Experimental Chemotherapy, vol. 3, pp. 21 7-270. Edited by R. H. Schnitzer and F. Hawking. New York: Academic Press.

SebeK, O. K. (1967). Polymyxins and circulin. In Antibiotics, vol. I, Mode of Action, pp. 142-152. Edited by D. Gottlieb and P. D. Shaw. Berlin: Springer Verlag.

YUDKIN, J. (1953). Origin of acquired drug resistance in bacteria. Nature, London 171, 541-546. 